\title{
Research on the Influence of Non-Stationary and Non-Gaussian Random Excitation on Structural Response Kurtosis
}

\section{Xin Zeng}

National University of Defense Technology https://orcid.org/0000-0003-2236-9776

Yu Jiang ( $\nabla$ jiangyu@nudt.edu.cn )

National University of Defense Technology https://orcid.org/0000-0003-0398-2938

Wuyang Lei

National University of Defense Technology

Zhengwei Fan

National University of Defense Technology

Original Article

Keywords: Non-Stationary, Non-Gaussian, Structural response kurtosis, Random vibration

Posted Date: July 9th, 2021

DOI: https://doi.org/10.21203/rs.3.rs-683449/v1

License: (c) (i) This work is licensed under a Creative Commons Attribution 4.0 International License.

Read Full License 


\section{Title page}

\section{Research on the Influence of Non-Stationary and Non-Gaussian Random Excitation on Structural Response Kurtosis}

Xin Zeng, born in 1991, is currently an engineer at National University of Defense Technology. He received his master degree from National University of Defense Technology, China, in 2021. His research interests include structural vibration fatigue.

Tel: +86-18053865820; E-mail: zengxin18@nudt.edu.cn

Yu Jiang, born in 1977, is currently a professor and a PhD candidate supervisor at Laboratory of Science and Technology on Integrated Logistics Support, National University of Defense Technology, China. His research interests include structural dynamic reliability, structural vibration fatigue and Structural health monitoring.

Tel: +86-13873190709; E-mail: jiangyu@nudt.edu.cn

Wu-Yang Lei, born in 1997, is currently a master candidate at Laboratory of Science and Technology on Integrated Logistics Support, National University of Defense Technology, China.

E-mail: leiwuyang@nudt.edu.cn

Zheng-Wei Fan, born in 1993, is currently a PhD candidate at Laboratory of Science and Technology on Integrated Logistics Support, National University of Defense Technology, China.

E-mail: fanzhengwei15@nudt.edu.cn

\section{Corresponding author: Yu Jiang E-mail: jiangyu@nudt.edu.cn}




\title{
Research on the Influence of Non-Stationary and Non-Gaussian Random Excitation on Structural Response Kurtosis
}

\author{
Xin Zeng ${ }^{1,2} \cdot$ Yu Jiang ${ }^{1,2}$ •Wu-Yang Lei ${ }^{1,2}$ - Zheng-Wei Fan ${ }^{1,2}$
}

Received June xx, 201x; revised February xx, 201x; accepted March xx, 201x

(C) Chinese Mechanical Engineering Society and Springer-Verlag Berlin Heidelberg 2017

\begin{abstract}
The stationary gaussian hypothesis is usually used to estimate the vibration fatigue life of structures. However, in actual engineering, the dynamic response of the structure usually exhibits non-stationary and non-gaussian, especially under harsh working condition or changing environment. The structural vibration fatigue life is closely related to the dynamic response characteristics, especially with respect to the structural response kurtosis used to characterize the non-Gaussian characteristics. In this paper, the influence of non-stationary and non-Gaussian random excitation on structural response kurtosis was studied by means of simulation and experiment. Firstly, by the means of simulating, the transmission law of excitation-response kurtosis was studied from three aspects, including system damping ratio, excitation frequency bandwidth, and excitation non-stationary characteristics. Then, the response kurtosis law was verified by the test results of cantilever vibration stress response. The results show that when the excitation is a stationary gaussian random load, the damping ratio and the excitation frequency bandwidth have no effect on the response kurtosis, and the response is approximately Gaussian distribution. When the excitation is stationary non-gaussian and non-stationary non-gaussian random load, if the damping ratio of the system is large, the response kurtosis is mainly affected by the damping ratio; If the damping ratio of the system is small, the frequency bandwidth and

Yu Jiang

jiangyu@nudt.edu.cn

1 College of Intelligence Science and Technology, National University of Defense Technology, Changsha 410073, China

2 Laboratory of Science and Technology on Integrated Logistics Support, National University of Defense Technology, Changsha 410073, China
\end{abstract}

non-stationarity of the excitation have significant effects on the response kurtosis. The research results can provide support for predicting the vibration response and fatigue life of engineering structures under complex non-stationary non-gaussian random loads.

Keywords: Non-Stationary • Non-Gaussian • Structural response kurtosis $\bullet$ Random vibration

\section{Introduction}

Vibration refers to the reciprocating motion of the structure near its equilibrium position. Any mechanical system may vibrate as long as it has elasticity and inertia. The influence of vibration environment and system dynamic characteristics on system response should be considered comprehensively in structural vibration response analysis. In engineering, it is assumed that the vibration environment is stationary gaussian (S-G), but many structural components are subjected to non-stationary non-gaussian (NS-NG) random loads in actual operation. Especially in vehicle transportation, marine equipment, aerospace and other fields, a large number of vibration fatigue failure problems have fully demonstrated that it is of great significance to carry out special research on structural vibration response under non-stationary and non-gaussian random excitation [1-4]. In addition, from the perspective of environmental test standards [5-7], some countries have gradually paid attention to the difference between non-stationary non-gaussian load and stationary Gaussian load on structural reliability and fatigue life. 
Based on recent work [8-10], the non-gaussian characteristics of structural stress response is the key problem to determine the random vibration fatigue life, so the kurtosis transfer law between excitation and response should be analyzed in random vibration response analysis.

Many scholars have studied this problem. Rizzi [11] studied the response laws of different non-gaussian excitation systems, but the research was limited to stationary non-gaussian processes. $\mathrm{Wu}$ [12] studied the acceleration response of the cantilever beam structure under the vibration excitation of ultra-gaussian, which did not involve the effect of system damping ratio. Morikawa et al. [13] proposed an extreme response statistics estimation method of single degrees of freedom system under the action of non-stationary gaussian white noise. Based on Hermite transformation model, Gao [14] researched the non-gaussian extremum response of offshore structures, and proposed the spectral analysis method of modal coupling. Quan [15] carried out wind tunnel test to study the probability density distribution characteristics of cross wind acceleration response. Based on the root mean square (RMS)-cycles of the acceleration, Wang [16] studied the non-gaussian acceleration random vibration model, but did not consider the effect of non-gaussian excitation with different bandwidth on the response. $\mathrm{Su}$ [17] researched the response of non-stationary fluctuating wind field to buffeting of bridge structure. Scott Karen [18] studied the application of Karhunen-Loeve extended method in solving the response of multi degree of freedom system under non-stationary gaussian vibration load. In recent years, Capponi and Palmieri et al. [19-20] have researched the non-stationary and non-gaussian vibration fatigue, but the coupling effect between non-stationary and non-gaussian, as well as the influence of random excitation bandwidth and structure characteristics on the response non-gaussian characteristics have not been considered. Yang [21] discussed the non-gaussian response process of automobile suspension spring and the vibration fatigue life of parts. Based on the explicit time domain method, Helu Yu [22] analyzed the non-stationary random vibration of vehicle bridge system excited by random track irregularity.

From the above research status, the influence of the non-stationary non-gaussian characteristics of random excitation and dynamic characteristics on the response kurtosis of the system have not been studied systematically. The theoretical background of the random process is introduced in Section 2. Taking the single degree of freedom system as the research object, the influence of non-stationary non-gaussian random excitation on the response kurtosis is researched based on simulation in
Section 3. The applicability of the kurtosis transfer law between excitation and response is verified by experiments in Section 4.

\section{Theoretical Background}

Although random vibration can not be described by the deterministic function of time, it has certain statistical regularity on the whole, so it can be described by statistical method [23]. According to whether the overall statistical characteristics change with time, the stochastic process can be divided into stationary random process and non-stationary random process. The following two aspects are the representation of stationary random process and the solution of non-stationary index.

\subsection{Stationary Random Process}

For stationary random processes $x(t)$, mean value $\mu_{x}$ standard deviation $\sigma_{x}$ root mean square (RMS) $\psi_{x}$ are used to characterize in time domain. Where covers both static and dynamic components of random vibration amplitude, the root mean square value $\psi_{x}$ is often used to represent the magnitude of signal energy.

$$
\begin{gathered}
\mu_{x}=E[x(t)] \\
\sigma_{x}=\sqrt{E\left[\left(x(t)-\mu_{x}\right)^{2}\right]} \\
\psi_{x}=\sqrt{E\left[x^{2}(t)\right]}
\end{gathered}
$$

Where $E(\cdot)$ denotes mathematical expectation. The autocorrelation function $R_{x x}(t, t+\tau)$ represent as the mathematical expectation of $[x(t) \cdot x(t+\tau)]$. For stationary random processes with ergodic states, the autocorrelation function $R_{x x}(t, t+\tau)$ is only concerned with time difference $\tau$.

$$
\begin{gathered}
R_{x x}(t, t+\tau)=E[x(t) \cdot x(t+\tau)], \\
R_{x x}(t, \tau)=R_{x x}(\tau)=\lim _{T \rightarrow \infty} \frac{1}{T} \int_{0}^{T} x(t) \cdot x(t+\tau) \mathrm{d} t,
\end{gathered}
$$

At the same time, the power spectral density (PSD) $S_{x x}(f)$ is usually used to describe stationary random processes in frequency domain, which just constitutes a pair of Fourier transform with the autocorrelation function $R_{x x}(t, t+\tau)$.

$$
S_{x x}(f)=\frac{1}{2 \pi} \int_{-\infty}^{+\infty} R_{x x}(\tau) \mathrm{e}^{-\mathrm{i} 2 \pi f \tau} \mathrm{d} \tau
$$




$$
R_{x x}(\tau)=\int_{-\infty}^{+\infty} 2 \pi S_{x x}(f) \mathrm{e}^{\mathrm{i} 2 \pi f \tau} \mathrm{d} f
$$

According to the probability density distribution of amplitude, random process can be divided into gaussian random process and non-gaussian random process. The probability density function $p(x)$ of gaussian distribution satisfies the form of Eq. (8). The $i$-order central moment $M_{i}$ can describe the numerical characteristics of probability density function of random process.

$$
\begin{aligned}
& p(x)=\frac{1}{\sqrt{2 \pi} \sigma_{x}} \exp \left[-\frac{\left(x-\mu_{x}\right)^{2}}{2 \sigma_{x}^{2}}\right] \\
& M_{i}=\int_{-\infty}^{\infty}\left[\left(x(t)-\mu_{x}\right)^{i}\right] \cdot p(x, t) \mathrm{d} x
\end{aligned}
$$

Generally, the characteristics of non-gaussian probability density distribution were described by kurtosis $K_{\mathrm{u}}$ (Eq. (10))and skewness $S_{\mathrm{k}}$ (Eq. (11)). Skewness $S_{\mathrm{k}}$ characterizes the asymmetry of probability density function curve, $S_{\mathrm{k}} \neq 0$ means that it does not obey symmetric distribution. Kurtosis $K_{\mathrm{u}}$ represents the sharpness of the peak and the width of the tail of the probability density function curve. $K_{\mathrm{u}}>3$ means that the signal is denser near the mean value and the probability of extremum is higher, which is compared with gaussian distribution [24].

$$
\begin{gathered}
K_{\mathrm{u}}=\frac{M_{4}}{M_{2}^{2}}=\frac{M_{4}}{\sigma^{4}} \\
S_{\mathrm{k}}=\frac{M_{3}}{M_{2}^{3 / 2}}=\frac{M_{3}}{\sigma^{3}}
\end{gathered}
$$

\subsection{Non-Stationary Index}

Although there is no unified and clear quantitative representation theory for non-stationary random processes, the non-stationary type of 'root mean square value' can be quantitatively characterized. The 'Run-Test', which belong to nonparametric statistical test method, can be used to check whether the sequence containing only two values conforms to the random hypothesis, and identify the process non-stationary without assuming that the tested data obey a specific probability distribution [25].

Firstly, the analysis signal is divided according to the same time window width. Afterwards, the change of the same statistical characteristics relative to the whole signal is calculated for each time window. According to the relationship between the statistical characteristics of the window signal and the test conditions, a value is assigned to each window. For example, each observation is classified into one of two categories and is represented by the symbol '+' or '-'. The total number of observations was $N$. Firstly, the number of ' + ' $N_{1}$ and the number of '-' $N_{2}$ were calculated. Then, the total 'run' number $r$ was evaluated. Finally, the nonstationary index $\gamma$ was solved. For a large sample, which both $N_{1}$ and $N_{2}$ are greater than 10 , the number of "run" $r$ is a random variable with normal distribution. Therefore, the normal distribution random variable $Z$ can be calculated as below [26].

$$
Z=\frac{r-\mu_{\mathrm{r}}}{\sigma_{\mathrm{r}}}
$$

Where mean $\mu_{\mathrm{r}}$ and variance $\sigma_{\mathrm{r}}^{2}$ can be represented by $N_{1}$ and $N_{2}$.

$$
\begin{gathered}
\mu_{\mathrm{r}}=\frac{2 N_{1} N_{2}}{N}+1 \\
\sigma_{\mathrm{r}}^{2}=\frac{2 N_{1} N_{2}\left(2 N_{1} N_{2}-N\right)}{N^{2}(N-1)}
\end{gathered}
$$

In order to keep the process stationary, the "run" number $r$ must fall at the level of significance $\alpha$ within the confidence interval determined as below.

$$
\mu_{\mathrm{r}}-Z_{1-\alpha / 2} \sigma_{\mathrm{r}} \leq r \leq \mu_{\mathrm{r}}+Z_{\alpha / 2} \sigma_{\mathrm{r}}
$$

When taking into consideration of significance level $\alpha=5 \%, Z_{1-\alpha / 2}=Z_{\alpha / 2} \approx 2$. Consequently, if the 'run' number $r$ falls within the confidence interval, the signal can be considered to be stationary, otherwise the signal is not. Then, the non-stationary index $\gamma$ can be calculated.

$$
\gamma=\left|\frac{r-\mu_{r}}{\sigma_{r}}\right|
$$

When $\gamma$ is close to 0 , it represents a stationary process. And considering the significance level $\alpha=5 \%$, when $0 \leq \gamma \leq 2$, it can be considered as a stationary process, when $\gamma>2$, it can not be considered as a stationary process [27].

The non-stationary index is highly sensitive to the time window width. Generally, it is required to calculate the root mean square value of the vibration process in a relatively narrow time window. The appropriate time window width is related to the impulse response period of the system, which can be defined as the time when the 
amplitude decreases to $10 \%$ of the initial value [28]. Since vibration fatigue research is mainly focused on specific systems, therefore the criterion can be used to measure the non-stationary index of excitation and response at the same time. The optimal window width $T_{\mathrm{w}}$ is expressed as,

$$
T_{\mathrm{w}} \approx \frac{\ln 10}{2 \pi \zeta f_{n}} \approx \frac{0.37}{\zeta f_{\mathrm{n}}}
$$

Where $\zeta$ and $f_{\mathrm{n}}$ represents the damping ratio and natural frequency of the system respectively.

\section{Simulation Analysis of Non-Stationary Non-Gaussian Random Vibration Response}

In this section, the amplitude modulation method of beta distribution random number was used to simulate non-stationary non-gaussian random signal as system excitation [27]. Taking a single degree-of-freedom mass-spring-damping system as an example, assumed that the mass $m=1 \mathrm{Kg}$ and the natural frequency $f_{\mathrm{n}}=200 \mathrm{~Hz}$ remain unchanged, the displacement response was obtained by applying displacement excitation, the effects of system damping ratio, excitation frequency bandwidth and non-stationary excitation signal on the transmission law of excitation response kurtosis have been investigated.

\subsection{Simulation Model}

As shown in Figure 1, a single degree-of-freedom (SDOF) system model under the foundation excitation is established, which consists of base, damping, spring and mass block.

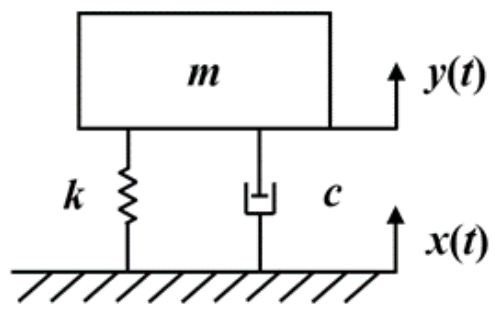

Figure 1 SDOF mass-spring-damping system model

The inertial force is opposite to the acceleration direction, $f_{\mathrm{I}}=-m \ddot{y}$. The damping force is opposite to the velocity direction, $f_{\mathrm{d}}=-c \dot{y}$. When the spring stiffness satisfies the linear condition, which elastic force $f_{\mathrm{s}}=-k y$, the single degree of freedom system can be simplified as a linear model, which was expressed in differential equation of motion as below.

$$
m \ddot{y}(t)+c \dot{y}(t)+k y(t)=c \dot{x}(t)+k x(t)
$$

Moreover, Eq. (18) can be indicated like:

$$
\ddot{y}(t)+4 \pi f \zeta \dot{y}(t)+4 \pi^{2} f^{2} y(t)=4 \pi f \zeta \dot{x}(t)+4 \pi^{2} f^{2} x(t)
$$

Where $\zeta$ and $f$ represent the damping ratio and natural frequency of SDOF system respectively.

$$
\begin{aligned}
& \zeta=\frac{c}{2 \sqrt{m k}} \\
& f=\frac{1}{2 \pi} \sqrt{\frac{k}{m}}
\end{aligned}
$$

\subsection{Influence of Damping Ratio on Response Kurtosis}

According to the resonance frequency of the system, different random excitation signals were generated by flat displacement power spectrum PSD1 (Figure 2).

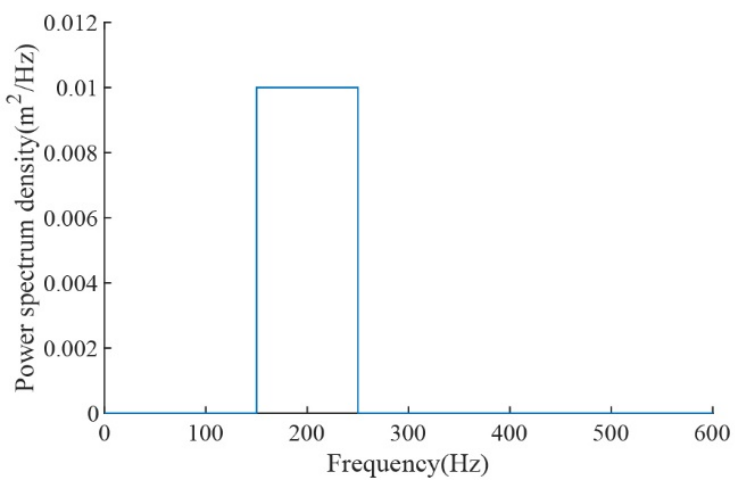

Figure 2 Displacement power spectral density under random excitation (PSD1)

Firstly, as shown in Figure 3, under different damping ratio, the responses kurtosis of stationary gaussian random excitation were around 3 , which were generally close to gaussian distribution. Secondly, as shown in Figure 4, the system responses of stationary non-gaussian random excitation were not necessarily gaussian distribution, but depend on the damping ratio $\zeta$. When $\zeta \leqslant 0.01$, the response was close to gaussian distribution. When the damping ratio $\zeta$ increases, the response tended to be non-gaussian distribution. 


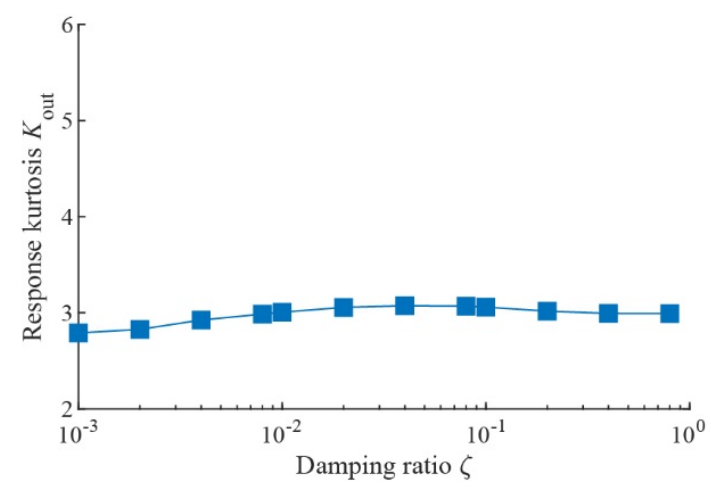

Figure 3 Kurtosis of system response under stationary gaussian random excitation and different damping ratio

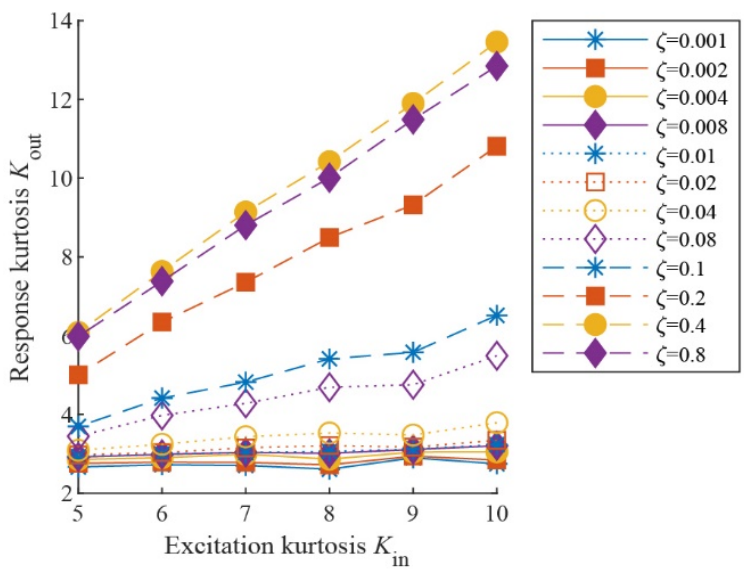

Figure 4 Under stationary non-gaussian random excitation, kurtosis of system response with different damping ratio

\subsection{Influence of Excitation Frequency Bandwidth on Response Kurtosis}

According to the PSD1 (Figure 2), keep the power spectral density unchanged, taking the natural frequency of the system $\left(f_{\mathrm{n}}=200 \mathrm{~Hz}\right)$ as the center frequency, four different frequency bandwidths were considered (Table 1).

Table 1 Four kinds of random excitation signals with different PSD bandwidth

\begin{tabular}{clll}
\hline NO. & Frequency range/(Hz) & Bandwidth $/(\mathrm{Hz})$ & PSD level $/\left(\mathrm{m}^{2} / \mathrm{Hz}\right)$ \\
\hline PSD1 & $150-250$ & 100 & 0.01 \\
PSD2 & $163.5-237.5$ & 75 & 0.01 \\
PSD3 & $175-225$ & 50 & 0.01 \\
PSD4 & $187.5-212.5$ & 25 & 0.01 \\
\hline
\end{tabular}

Firstly, as shown in Figure 5, the responses kurtosis was approximately 3, which was gaussian distribution. When the damping ratio was very low $(\zeta=0.001)$, the responses kurtosis was slightly different under different frequency bandwidth. However, when the damping ratio of the system increased gradually, the PSD frequency bandwidth of the excitation signal had little effect on the response kurtosis.

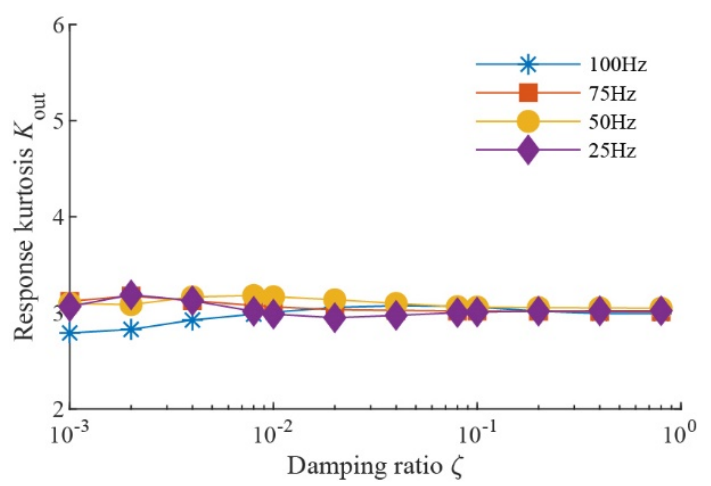

Figure 5 Under stationary gaussian random excitation, kurtosis of system response with different damping ratio and frequency bandwidth

Secondly, based on the results in Section 3.2 (Figure 4), when the damping ratio was low, the response kurtosis under stationary non-gaussian random excitation tended to be gaussian distribution. Therefore, two different damping ratios $(0.001,0.1)$ have been considered. For low damping ratio $(\zeta=0.001)$, the responses kurtosis was still close to 3 (Figure 6(a)), which were gaussian distribution. For high damping ratio $(\zeta=0.1)$, the responses kurtosis was close to the excitation kurtosis as the excitation frequency bandwidth decreases (Figure 6(b)). Therefore, only when the damping ratio is relatively high, the frequency bandwidth of the excitation signal will affect the response kurtosis.

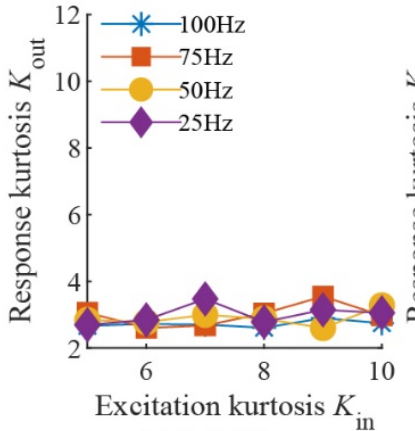

(a) $\zeta=0.001$

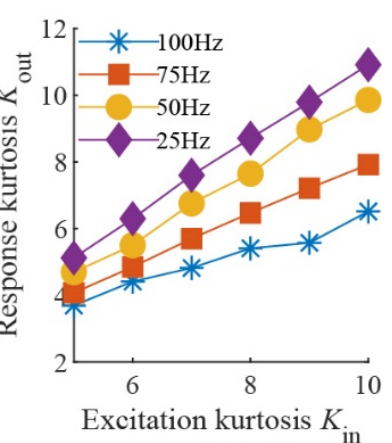

(b) $\zeta=0.1$
Figure 6 Under stationary non-gaussian random excitation, kurtosis of system response with different frequency bandwidth and two damping ratio $(0.001,0.1)$ 


\subsection{Influence of Excitation Non-stationary on \\ Response Kurtosis}

Four groups of non-stationary non-gaussian random excitation signals with different non-stationary degrees were generated according to the PSD1 with $100 \mathrm{~Hz}$ bandwidth (Figure 2). According to the time window $T_{\mathrm{w}}=$ $0.1 \mathrm{~s}$, the non-stationary index of each group of signals were obtained by 'Run-Test' method. According to the plots in Figure 7 , it can be seen that the non-stationary index of signals from group A to D increases gradually.

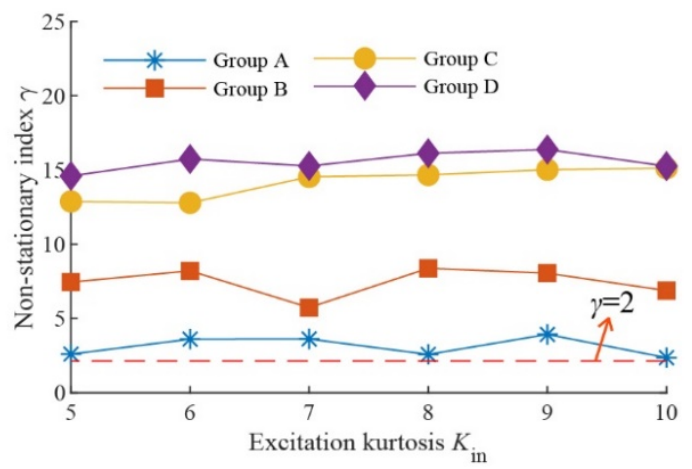

Figure 7 Non-stationary index test results for group A-D

Firstly, taking the non-stationary group D as an example, the response kurtosis of non-stationary non-gaussian random excitation under different damping ratios were studied. As shown in Figure 8, the response kurtosis was greater than 4, which was obviously non-gaussian distribution. Compared with the stationary non-gaussian excitation (Figure 4), when $\zeta>0.002$, the response kurtosis of non-stationary non-gaussian excitation were greater than that of stationary non-gaussian excitation. When the damping ratio increased, the response kurtosis was approximately equal to the excitation kurtosis.

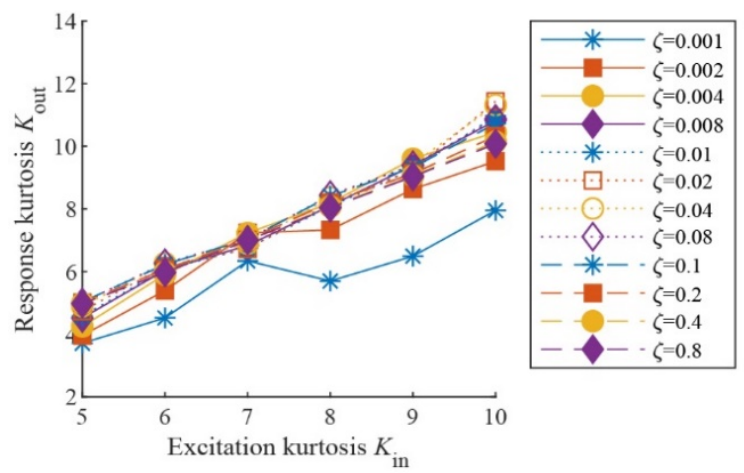

Figure 8 Under non-stationary non-gaussian random excitation, kurtosis of system response with different damping ratio
Secondly, two different damping ratios $(0.001,0.1)$ were considered for group A-D. As shown in Figure 9(a), for low damping ratio $(\zeta=0.001)$, the lower the non-stationary index of the excitation signal (group A), the closer the response kurtosis were to Gaussian distribution. As shown in Figure 9(b), for high damping ratio $(\zeta=0.1)$, the response kurtosis was approximately equal to the excitation kurtosis.

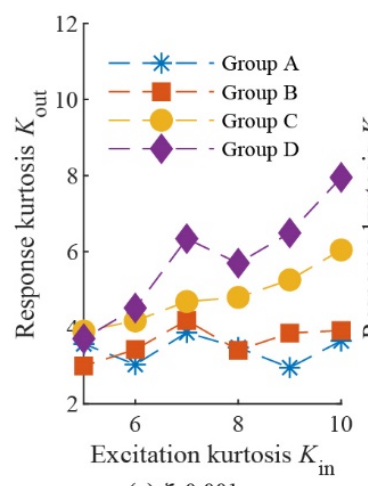

(a) $\zeta=0.001$

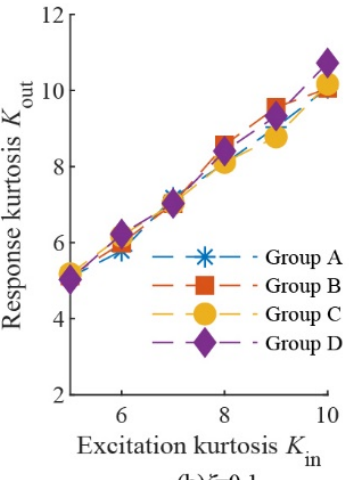

(b) $\zeta=0.1$
Figure 9 Under non-stationary non-gaussian random excitation, kurtosis of system response with different degrees of non-stationary and two damping ratio $(0.001,0.1)$

Thirdly, based on four different frequency bandwidths (Table 1), non-stationary non-gaussian random excitation signals with similar non-stationary index were generated. Through the 'Run-Test', the non-stationary index of each group was obtained. As shown in Figure 10, the non-stationary index of each group was all in the range of $[2,4]$.

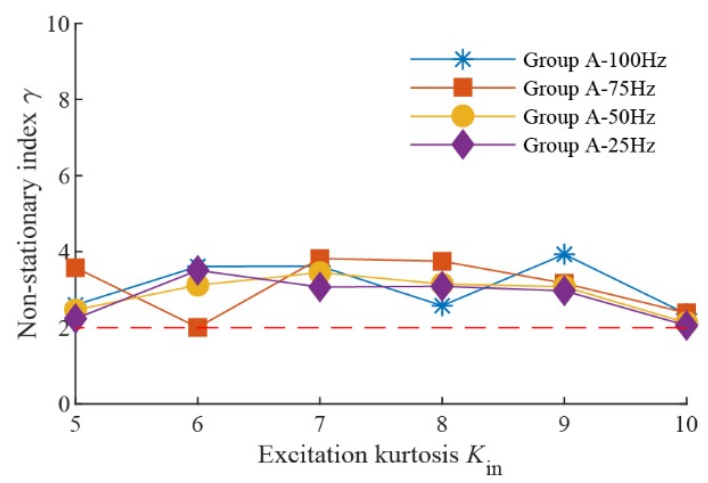

Figure 10 Non-stationary index test results for different frequency bandwidth

Three damping ratios $(0.001,0.01,0.1)$ have been considered. As shown in Figure 11(a), when the damping ratio was low $(\zeta=0.001)$, the frequency bandwidth of the 
excitation signal had little effect on the response kurtosis, which is close to 3 . When the damping ratio was moderate $(\zeta=0.01)$, as shown in Figure 11(b), the frequency bandwidth has an unordered effect on the response kurtosis, which were non-gaussian distribution. When the damping ratio was high $(\zeta=0.1)$, as shown in Figure 11(c), the frequency bandwidth of the excitation signal had little effect on the response kurtosis, which were approximately equal to the excitation kurtosis.

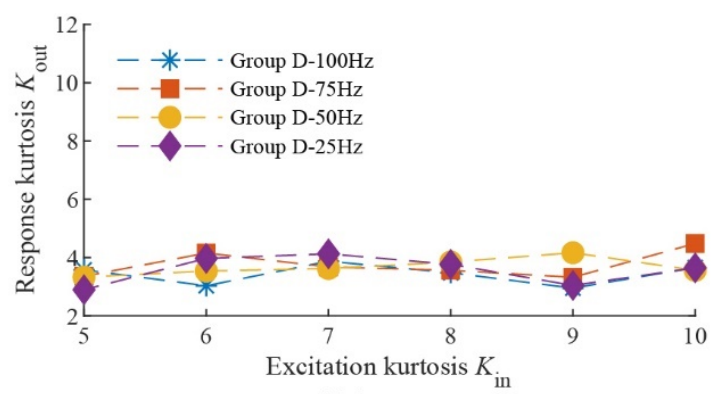
(a) $\zeta=0.001$

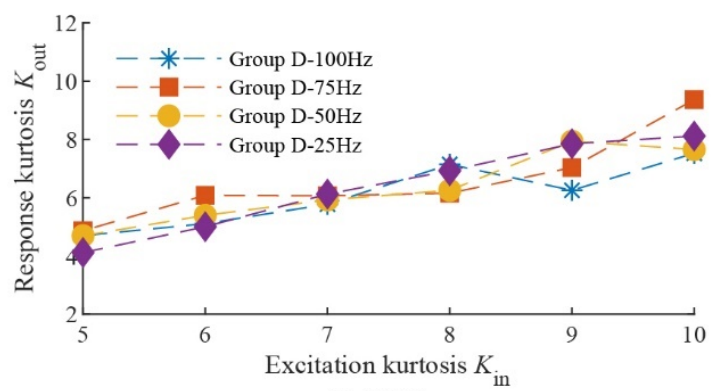
(b) $\zeta=0.01$

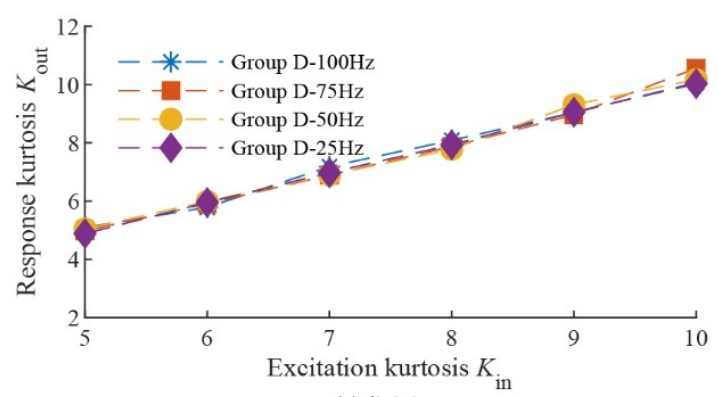

(c) $\zeta=0.1$

Figure 11 Under non-stationary non-gaussian random excitation, kurtosis of system response with different frequency bandwidth and three damping ratios $(0.001,0.01,0.1)$

\section{Experimental Verification}

\subsection{Test Preparation}

The V-notch cantilever beam was selected in the test, and the geometric dimensions are shown in Figure 12. The material of specimen was aluminum alloy 6061-T6, of what maximum tensile strength is $310.26 \mathrm{MPa}$.

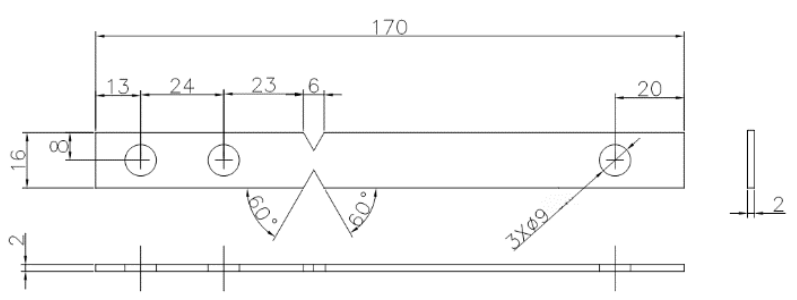

Figure 12 Structure and size of specimen (unit: $\mathrm{mm}$ )

The shaking table test adopts the loading mode of foundation excitation. In order to meet the requirement of four specimens in each vibration test, it was necessary to design a fixture to hold four specimens in the same way. The fixture consisted of a base and two battens. The base was fixed on the shaking table by six bolts, and each specimen was fixed between the base and the battens by two bolts. For the sake of accelerating the fatigue process of the specimen, the counterweight bolt was installed at the through hole of the cantilever end of each specimen. The schematic diagram of counterweight bolt installation and signal acquisition channel is shown in Figure 13. The first-order natural frequency of specimen was $26.01 \mathrm{~Hz}$, which was carried out by the sine sweep frequency test.

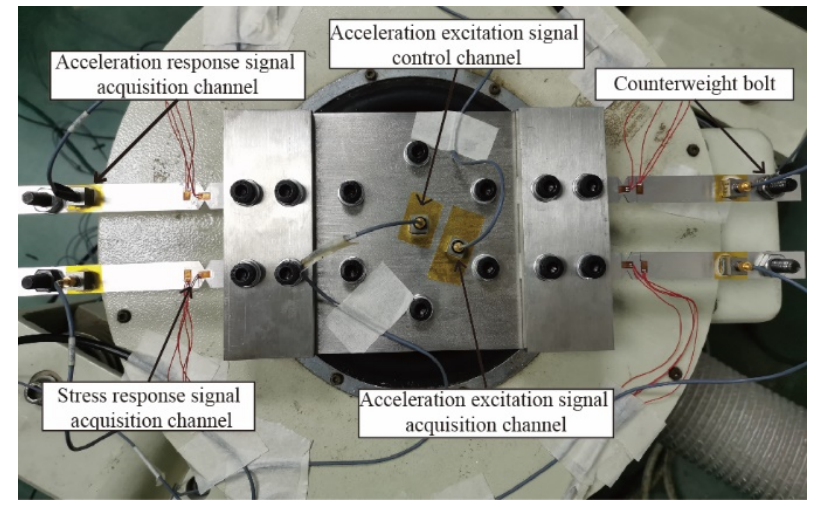

Figure 13 Schematic diagram of signal acquisition channel

\subsection{Test Profile}

In order to research the influence of non-stationary and non-gaussian characteristics of random excitation on the kurtosis of vibration stress response, five groups of tests (A-E) have been designed. The frequency range of each test profile includes the first-order natural frequency to ensure the resonance of the specimen in the vibration test.

Group A: Based on the stationary gaussian (S-G) random excitation $\mathrm{A} 1$, the $\mathrm{PSD}$ level was changed, as shown in Table 2. 
Table 2 Profile of test group A

\begin{tabular}{llllll}
\hline NO. & Type & $\begin{array}{c}\text { Bandwidth } \\
/(\mathrm{Hz})\end{array}$ & $\begin{array}{c}\text { PSD level } \\
/\left(\mathrm{g}^{2} / \mathrm{Hz}\right)\end{array}$ & $\begin{array}{c}\text { RMS } \\
/(\mathrm{g})\end{array}$ & Kurtosis \\
\hline A1 & S-G & $10-110$ & 0.02 & 1.41 & 3 \\
A2 & S-G & $10-110$ & 0.04 & 2.00 & 3 \\
A3 & S-G & $10-110$ & 0.05 & 2.24 & 3 \\
A4 & S-G & $10-110$ & 0.06 & 2.45 & 3 \\
A5 & S-G & $10-110$ & 0.08 & 2.83 & 3 \\
\hline
\end{tabular}

Group B: Based on the stationary gaussian random excitation B1(A3), the kurtosis value was changed, as shown in Table 3.

Table 3 Profile of test group B

\begin{tabular}{llllll}
\hline NO. & Type & $\begin{array}{c}\text { Bandwidth } \\
/(\mathrm{Hz})\end{array}$ & $\begin{array}{c}\text { PSD level } \\
/\left(\mathrm{g}^{2} / \mathrm{Hz}\right)\end{array}$ & $\begin{array}{c}\text { RMS } \\
/(\mathrm{g})\end{array}$ & Kurtosis \\
\hline B1(A3) & S-G & $10-110$ & 0.05 & 2.24 & 3 \\
B2 & S-NG & $10-110$ & 0.05 & 2.24 & 5 \\
B3 & S-NG & $10-110$ & 0.05 & 2.24 & 7 \\
\hline
\end{tabular}

Group C: Based on the stationary non-gaussian (S-NG) random excitation $\mathrm{C} 1$ (B2), the frequency bandwidth was changed, as shown in Table 4.

Table 4 Profile of test group C

\begin{tabular}{llllll}
\hline \multicolumn{1}{c}{ NO. } & Type & $\begin{array}{c}\text { Bandwidth } \\
/(\mathrm{Hz})\end{array}$ & $\begin{array}{c}\text { PSD level } \\
/\left(\mathrm{g}^{2} / \mathrm{Hz}\right)\end{array}$ & $\begin{array}{c}\text { RMS } \\
/(\mathrm{g})\end{array}$ & Kurtosis \\
\hline C1(A3) & S-NG & $10-110$ & 0.05 & 2.24 & 5 \\
C2 & S-NG & $10-85$ & 0.05 & 1.94 & 5 \\
C3 & S-NG & $10-60$ & 0.05 & 1.58 & 5 \\
C4 & S-NG & $20-30$ & 0.05 & 0.71 & 5 \\
\hline
\end{tabular}

Table 5 Profile of test group D

\begin{tabular}{llllll}
\hline \multicolumn{1}{c}{ NO. } & Type & $\begin{array}{c}\text { Band- } \\
\text { width } \\
/(\mathrm{Hz})\end{array}$ & $\begin{array}{c}\text { PSD level } \\
\text { combination } \\
/\left(\mathrm{g}^{2} / \mathrm{Hz}\right)\end{array}$ & $\begin{array}{c}\text { RMS } \\
/(\mathrm{g})\end{array}$ & Kurtosis \\
\hline D1(A3,B1) & S-G & $10-110$ & 0.05 & 2.24 & 3 \\
D2 & NS-G & $10-110$ & $0.06-0.04$ & 2.24 & 3 \\
D3 & NS-G & $10-110$ & $0.07-0.03$ & 2.24 & 3 \\
D4 & NS-G & $10-110$ & $0.08-0.02$ & 2.24 & 3 \\
\hline
\end{tabular}

Group D: Based on the stationary gaussian random excitation D1(A3, B1), keeping the frequency bandwidth unchanged, permutation and combination in time domain were carried out with different PSD levels, as shown in Table 5. Through time domain and frequency domain analysis of group D, as shown in Figure 14, it can be seen that although D1 to D4 show different non-stationary characteristics in time domain, they all have the same shape of power spectral density in frequency domain, and the overall root mean square values were equal.

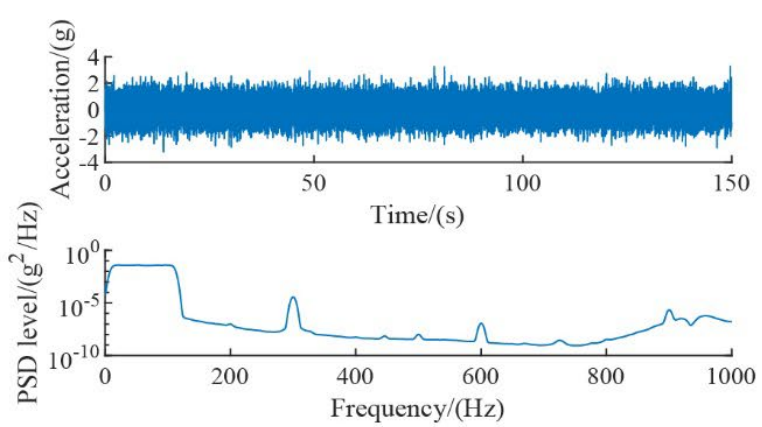

(a) Stationary gaussian D1(A3, B1)

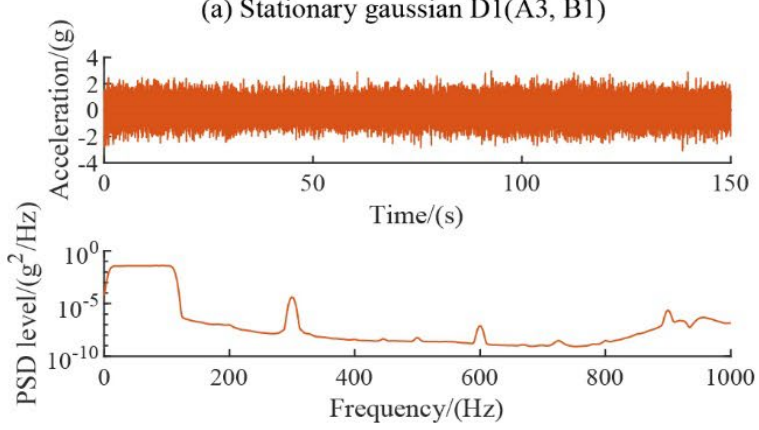

(b) Non-stationary gaussian D2

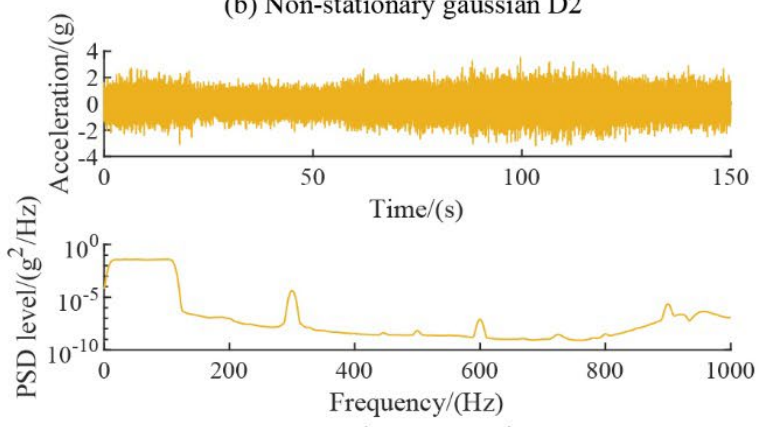

(c) Non-stationary gaussian D3

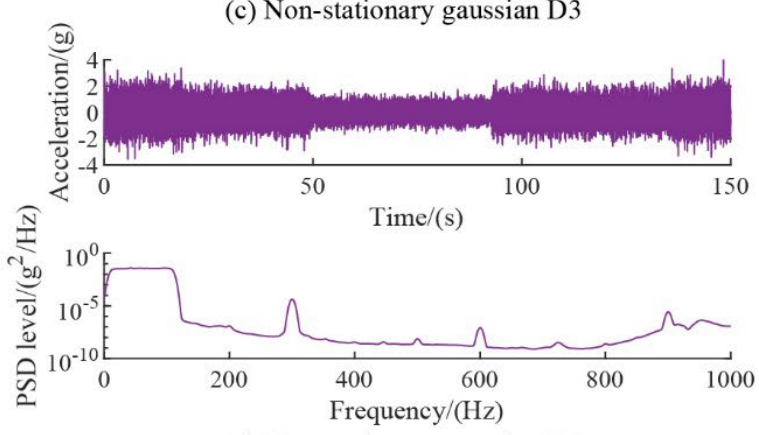

(d) Non-stationary gaussian D4

Figure 14 Time domain and frequency domain diagrams of acceleration excitation for test group D

Table 6 Profile of test group D

\begin{tabular}{lllll}
\hline NO. & Type & $\begin{array}{c}\text { Bandwidth } \\
/(\mathrm{Hz})\end{array}$ & $\begin{array}{c}\text { PSD level combination } \\
/\left(\mathrm{g}^{2} / \mathrm{Hz}\right)\end{array}$ & Kurtosis \\
\hline E1(D3) & NS-G & $10-110$ & $0.07-0.03$ & 3 \\
E2 & NS-NG & $10-110$ & $0.07-0.03$ & 5 \\
E3 & NS-NG & $10-110$ & $0.07-0.03$ & 7 \\
\hline
\end{tabular}

Group E: Based on non-stationary gaussian (NS-G) 
random excitation E1(D3), kurtosis value was changed, as shown in Table 6.

\subsection{Analysis of Structural Stress Response Test Results}

The stress signals at the fracture notch under each test section have been collected and analyzed to verify the random vibration response law from the aspects of excitation / response non-stationary index, stress response RMS value, stress response kurtosis, etc. Since the maximum frequency of excitation is $110 \mathrm{~Hz}$, the sampling frequency is $500 \mathrm{~Hz}$ and the sampling time is $150 \mathrm{~s}$ according to Nyquist sampling law. Owning to the damping ratio of ordinary aluminum alloy plates is generally 0.01 to 0.03 [29], the damping ratio of specimens was assumed in this section $\zeta=0.015$. Thereupon, the width of non-stationary test time window $T_{\mathrm{w}}=1 \mathrm{~s}$ was determined, according to Eq. (17).

\subsubsection{Stress response test results of test group A}

From the non-stationary test results (Table 7), both the acceleration excitation and stress response of group $\mathrm{A}$ were stationary. The results of stress response are shown in Table 8. With the increase of PSD level, the kurtosis of stress responses was around 3, which were close to gaussian distribution. Moreover, there is a linear relationship between PSD magnitude and RMS value of stress response.

Table 7 Comparison of non-stationary test results of acceleration excitation and stress response in group A

\begin{tabular}{|c|c|c|c|c|c|c|c|}
\hline \multirow{2}{*}{ NO. } & \multirow{2}{*}{ Type } & \multirow{2}{*}{$\mathrm{Ku}$} & \multirow{2}{*}{$\begin{array}{l}\mathrm{Tw} \\
/(\mathrm{s})\end{array}$} & \multicolumn{2}{|c|}{ Acceleration } & \multicolumn{2}{|c|}{ Stress } \\
\hline & & & & Index $\gamma$ & Assess & Index $\gamma$ & Assess \\
\hline A1 & S-G & 3 & 1 & 0.80 & $\mathrm{~S}$ & 0.51 & $\mathrm{~S}$ \\
\hline $\mathrm{A} 2$ & S-G & 3 & 1 & 0.09 & $\mathrm{~S}$ & 0.89 & $\mathrm{~S}$ \\
\hline A3 & S-G & 3 & 1 & 0.44 & $\mathrm{~S}$ & 1.80 & $\mathrm{~S}$ \\
\hline A4 & S-G & 3 & 1 & 0.50 & $\mathrm{~S}$ & 1.37 & $\mathrm{~S}$ \\
\hline A5 & S-G & 3 & 1 & 0.14 & $\mathrm{~S}$ & 1.88 & S \\
\hline
\end{tabular}

Table 8 Comparison of stress response characteristics of test group A

\begin{tabular}{llllll}
\hline NO. & Type & $\begin{array}{c}\text { PSD level } \\
/\left(\mathrm{g}^{2} / \mathrm{Hz}\right)\end{array}$ & $\begin{array}{c}\text { Stress } \\
\text { RMS } /(\mathrm{MPa})\end{array}$ & $\begin{array}{c}\text { Stress } \\
\text { kurtosis }\end{array}$ & $\begin{array}{c}\text { Time } \\
\text { span/(s) }\end{array}$ \\
\hline A1 & S-G & 0.02 & 17.47 & 2.91 & 150 \\
A2 & S-G & 0.04 & 26.36 & 3.19 & 150 \\
A3 & S-G & 0.05 & 30.80 & 3.41 & 150 \\
A4 & S-G & 0.06 & 33.73 & 3.14 & 150 \\
A5 & S-G & 0.08 & 45.08 & 3.30 & 150 \\
\hline
\end{tabular}

\subsubsection{Stress response test results of test group $B$}

From the non-stationary test results (Table 9), both the acceleration excitation and stress response of group $B$ were stationary. The results of stress response are shown in Table 10. First, although the kurtosis of excitation was different, the RMS value of stress response were basically unchanged due to the same PSD level of excitation. Second, with the increase of the excitation kurtosis, the kurtosis of the stress response increases slightly, but it was still close to gaussian, indicating that the damping of the specimen was relatively small, which is consistent with the law in Figure 4.

Table 9 Comparison of non-stationary test results of acceleration excitation and stress response in group B

\begin{tabular}{llllllll}
\hline \multirow{2}{*}{ NO. } & \multirow{2}{*}{ Type } & \multirow{2}{*}{$K \mathrm{u}$} & \multirow{2}{*}{\begin{tabular}{c} 
Tw \\
\cline { 5 - 8 }
\end{tabular}} & & \multicolumn{2}{c}{ Acceleration } & \multicolumn{2}{c}{ Stress } \\
\cline { 5 - 8 } & & & Index $\gamma$ & Assess & Index $\gamma$ & Assess \\
\hline B1(A3) & S-G & 3 & 1 & 0.44 & $\mathrm{~S}$ & 1.80 & $\mathrm{~S}$ \\
B2 & S-NG & 5 & 1 & 1.56 & $\mathrm{~S}$ & 0.04 & $\mathrm{~S}$ \\
B3 & S-NG & 7 & 1 & 0.15 & $\mathrm{~S}$ & 0.44 & $\mathrm{~S}$ \\
\hline
\end{tabular}

Table 10 Comparison of stress response characteristics of test group B

\begin{tabular}{llllll}
\hline NO. & Type & $\begin{array}{c}\text { Acceleration } \\
\text { kurtosis }\end{array}$ & $\begin{array}{c}\text { Stress } \\
\text { RMS/(MPa) }\end{array}$ & $\begin{array}{c}\text { Stress } \\
\text { kurtosis }\end{array}$ & $\begin{array}{c}\text { Time } \\
\text { span/(s) }\end{array}$ \\
\hline B1(A3) & S-G & 3 & 30.80 & 3.41 & 150 \\
B2 & S-NG & 5 & 29.59 & 3.77 & 150 \\
B3 & S-NG & 7 & 30.90 & 3.83 & 150 \\
\hline
\end{tabular}

\subsubsection{Stress response test results of test group $C$}

From the non-stationary test results (Table 11), both the acceleration excitation and stress response of group $\mathrm{C}$ are stationary. The results of stress response are shown in Table 12. First, although the RMS value of acceleration excitation decreases, the RMS value of stress response basically remains unchanged, which indicates that the RMS value of stress response is mainly related to the level of the power spectral density curve of acceleration excitation at the first-order modal frequency of the specimen, However, it is not closely related to the frequency bandwidth of the excitation. Second, with the decrease of the excitation frequency bandwidth, the response kurtosis increases and approaches to the non-gaussian distribution, which is consistent with the law in Figure 6.

Table 11 Comparison of non-stationary test results of acceleration excitation and stress response in group $\mathrm{C}$

\begin{tabular}{llllllll}
\hline \multirow{2}{*}{ NO. } & \multirow{2}{*}{ Type } & \multirow{2}{*}{$\mathrm{Ku}$} & \multirow{2}{*}{ Tw } & \multicolumn{2}{c}{ Acceleration } & \multicolumn{2}{c}{ Stress } \\
\cline { 5 - 8 } & & & (s) & $\operatorname{Index} \gamma$ & Assess & Index $\gamma$ & Assess \\
\hline C1(B2) & S-NG & 5 & 1 & 1.56 & $\mathrm{~S}$ & 0.04 & $\mathrm{~S}$ \\
C2 & S-NG & 5 & 1 & 0.66 & $\mathrm{~S}$ & 0.31 & $\mathrm{~S}$ \\
$\mathrm{C} 3$ & $\mathrm{~S}-\mathrm{NG}$ & 5 & 1 & 1.05 & $\mathrm{~S}$ & 0.86 & $\mathrm{~S}$ \\
$\mathrm{C} 4$ & $\mathrm{~S}-\mathrm{NG}$ & 5 & 1 & 0.78 & $\mathrm{~S}$ & 0.74 & $\mathrm{~S}$ \\
\hline
\end{tabular}


Table 12 Comparison of stress response characteristics of test group $\mathrm{C}$

\begin{tabular}{lllllll}
\hline NO. & Type & $\begin{array}{c}\text { Band- } \\
\text { width } \\
/(\mathrm{Hz})\end{array}$ & $\begin{array}{c}\text { Acceleration } \\
\text { RMS } \\
/(\mathrm{g})\end{array}$ & $\begin{array}{c}\text { Stress } \\
\text { RMS } \\
/(\mathrm{MPa})\end{array}$ & $\begin{array}{c}\text { Stress } \\
\text { kurtosis }\end{array}$ & $\begin{array}{c}\text { Time } \\
\text { span } \\
/(\mathrm{s})\end{array}$ \\
\hline C1(B2) & S-NG & $10-110$ & 2.24 & 29.59 & 3.77 & 150 \\
C2 & S-NG & $10-85$ & 1.94 & 30.75 & 3.81 & 150 \\
C3 & S-NG & $10-60$ & 1.58 & 30.45 & 4.06 & 150 \\
C4 & S-NG & $20-30$ & 0.71 & 29.21 & 4.62 & 150 \\
\hline
\end{tabular}

\subsubsection{Stress response test results of test group D}

From the non-stationary test results (Table 13), group D non-stationary acceleration excitation will lead to non-stationary stress response. The results of stress response are shown in Table 14. Firstly, with the increase of non-stationary index of excitation (RMS value fluctuation amplitude), the RMS value of stress response increased slightly. Secondly, the kurtosis of the stress response gradually increased, especially when the RMS fluctuation was very significant (D4). Thirdly, compared with the results of test group B (Table 10), the kurtosis of the stress response presents obvious non-gaussian characteristics.

Table 13 Comparison of non-stationary test results of acceleration excitation and stress response in group D

\begin{tabular}{|c|c|c|c|c|c|c|c|}
\hline \multirow[b]{2}{*}{ NO. } & \multirow[b]{2}{*}{ Type } & \multirow[b]{2}{*}{$\mathrm{Ku}$} & \multirow[b]{2}{*}{$\begin{array}{l}\text { Tw } \\
/(\mathrm{s})\end{array}$} & \multicolumn{2}{|c|}{ Acceleration } & \multicolumn{2}{|c|}{ Stress } \\
\hline & & & & $\begin{array}{c}\text { Index } \\
\gamma \\
\end{array}$ & Assess & $\begin{array}{c}\text { Index } \\
\gamma \\
\end{array}$ & Assess \\
\hline$\overline{D 1(A 3, B 1)}$ & S-G & 3 & 1 & 0.44 & $\bar{S}$ & 1.80 & $\mathrm{~S}$ \\
\hline $\mathrm{D} 2$ & NS-G & 3 & 1 & 5.73 & NS & 3.57 & NS \\
\hline D3 & NS-G & 3 & 1 & 6.39 & NS & 3.19 & NS \\
\hline D4 & NS-G & 3 & 1 & 7.99 & NS & 7.01 & NS \\
\hline
\end{tabular}

Table 14 Comparison of stress response characteristics of test group D

\begin{tabular}{llllll}
\hline NO. & Type & $\begin{array}{c}\text { PSD level } \\
\text { combination } \\
/\left(\mathrm{g}^{2} / \mathrm{Hz}\right)\end{array}$ & $\begin{array}{c}\text { Stress } \\
\text { RMS } \\
/(\mathrm{MPa})\end{array}$ & $\begin{array}{c}\text { Stress } \\
\text { kurtosis }\end{array}$ & $\begin{array}{c}\text { Time } \\
\text { span } \\
/(\mathrm{s})\end{array}$ \\
\hline D1(A3,B1) & S-G & 0.05 & 30.80 & 3.41 & 150 \\
D2 & NS-G & $0.06-0.04$ & 30.05 & 3.26 & 150 \\
D3 & NS-G & $0.07-0.03$ & 30.97 & 3.52 & 150 \\
D4 & NS-G & $0.08-0.02$ & 31.57 & 4.58 & 150 \\
\hline
\end{tabular}

\subsubsection{Stress response test results of test group $E$}

From the non-stationary test results (Table 15), group E non-stationary acceleration excitation will lead to non-stationary stress response. The stress response results are shown in Table 16. Firstly, when the kurtosis of non-stationary excitation increased, the RMS value of stress response were basically unchanged. Secondly, compared with the non-stationary gaussian random excitation E1(D3), the stress response kurtosis value of the non-stationary non-gaussian random excitation(E3) was higher, which is consistent with Figure 8.

Table 15 Comparison of non-stationary test results of acceleration excitation and stress response in group $\mathrm{E}$

\begin{tabular}{llllllll}
\hline \multirow{2}{*}{ NO. } & \multirow{2}{*}{ Type } & \multirow{2}{*}{$\mathrm{Ku}$} & \multirow{2}{*}{\begin{tabular}{l}
$\mathrm{Tw}$ \\
\cline { 5 - 8 }
\end{tabular}} & & \multicolumn{2}{c}{ Acceleration } & \multicolumn{2}{c}{ Stress } \\
\cline { 5 - 8 } & & & Index $\gamma$ & Assess & Index $\gamma$ & Assess \\
\hline E1(D3) & NS-G & 3 & 1 & 6.39 & $\mathrm{~S}$ & 3.19 & $\mathrm{~S}$ \\
E2 & NS-NG & 5 & 1 & 6.17 & NS & 4.75 & NS \\
E3 & NS-NG & 7 & 1 & 2.37 & NS & 4.40 & NS \\
\hline
\end{tabular}

Table 16 Comparison of stress response characteristics of test group E

\begin{tabular}{llllll}
\hline NO. & Type & $\begin{array}{c}\text { PSD level } \\
\text { combination } \\
/\left(\mathrm{g}^{2} / \mathrm{Hz}\right)\end{array}$ & $\begin{array}{c}\text { Stress } \\
\text { RMS } \\
/(\mathrm{MPa})\end{array}$ & $\begin{array}{c}\text { Stress } \\
\text { kurtosis }\end{array}$ & $\begin{array}{c}\text { Time } \\
\text { span } \\
/(\mathrm{s})\end{array}$ \\
\hline E1(D3) & NS-G & $0.07-0.03$ & 30.97 & 3.52 & 150 \\
E2 & NS-NG & $0.07-0.03$ & 29.68 & 3.96 & 150 \\
D3 & NS-NG & $0.07-0.03$ & 29.84 & 4.73 & 150 \\
\hline
\end{tabular}

\section{Conclusions}

The influence of non-stationary and non-gaussian characteristics of random vibration on structural vibration response were researched by means of simulation and experiment, which can provide support for predicting the vibration response and fatigue life of engineering structures under complex non-stationary and non-gaussian random loads. The main research conclusions are below:

(1) When the excitation is a stationary gaussian random load, the damping ratio and the excitation frequency bandwidth have no effect on the response kurtosis, and the response is approximately gaussian distribution.

(2) When the excitation is stationary non-gaussian and non-stationary non-gaussian random loads, if the damping ratio of the system is relatively high, the response kurtosis is mainly affected by the damping ratio,

(3) When the excitation is stationary non-gaussian and non-stationary non-gaussian random loads, if the damping ratio of the system is relatively low, the frequency bandwidth and non-stationarity of the excitation have significant effects on the response kurtosis.

The research results can provide support for predicting the vibration response and fatigue life of engineering structures under complex non-stationary and non-gaussian random loads.

\section{Declaration}




\section{Acknowledgements}

The authors sincerely thanks to Professor Junyong Tao and Professor Honggang Zhao of National University of Defense Technology for his critical discussion and reading during manuscript preparation.

\section{Funding}

Supported by National Natural Science Foundation of China (Grant No. 51875570).

\section{Availability of data and materials}

The datasets supporting the conclusions of this article are included within the article.

\section{Authors' contributions}

The author' contributions are as follows: Xin Zeng carried out the experiment research and wrote the manuscript, Yu Jiang reviewed and edited the manuscript, Wu-Yang Lei and Zheng-Wei Fan assisted with laboratory analyses and investigation. All authors read and approved the final manuscript.

\section{Competing interests}

The authors declare no competing financial interests.

\section{Consent for publication}

Not applicable

\section{Ethics approval and consent to participate}

Not applicable

\section{References}

[1] V Rouillard. Statistical models for non-stationary and non-gaussian road vehicle vibration. Engineering Letters, 2009, 17(4): 227-237.

[2] J H Liao. Simulation of non-stationary non-gaussian random process and application to seismic reliability of structures. Chongqing: Chongqing University, 2019. (in Chinese)

[3] H J Kim, B S Jang. Fatigue life prediction of ship and offshore structures under wide-banded non-gaussian random loading: part II: Extension to wide-banded non-gaussian random process. Applied Ocean Research, 2021, 106(102480).

[4] F Han, C Wang, A Hu. Numerical investigation of wave-induced vibrations and their effect on the fatigue damage of container ships. Ocean Engineering, 2017, 142(245-258).

[5] USA Department of Defense, Department of Defense Test Method Standard for Environment Engineering Considerations and Laboratory Test: MIL-STD-810H. 2019.

[6] UK Ministry of Defense, Defense Standard 00-35 Issue 4: Environmental Handbook for Defense Materiel: Part 3: Environment Test Method. 2006.

[7] GJB-150.16A. Laboratory Environment Test Method for Military Materiel-Part 16: Vibration Test. 2009. (in Chinese)

[8] Y Jiang, J Y Tao, H W Cheng. Non-gaussian random vibration fatigue analysis and test technology. Beijing: National Defense Industry Press, 2019. (in Chinese)

[9] H W Cheng. Research on fatigue life and reliability of structure under non-gaussian random load. Changsha: National University of Defense Technology, 2014. (in Chinese)

[10]M Shuang, B Song. Influence of non-gaussian wind load on fatigue damage of wind turbine structure. Journal of Harbin Institute of Technology, 2017, 49(12): 152-158. (in Chinese)

[11]Rizzi, Adam, Turner, Travis. On the response of a nonlinear structure to high kurtosis non-gaussian random loadings. NASA Technical Report, 2013.

[12]Y C Wu. Stress response and fatigue life analysis of typical structures under super-gaussian vibration excitation. Changsha: National University of Defense Technology, 2012. (in Chinese)

[13]Morikawa, Hitoshi1, Z Aspasia. Approximate representation of the statistics for extreme responses of single degree-of-freedom system excited by non-stationary processes. Probabilistic Engineering Mechanics, 2008, 2-3: 279-288.

[14]S Gao. Research on the non-gaussian response extremum and fatigue of marine engineering structures. Dalian: Dalian University of Technology, 2019. (in Chinese)

[15]Y Quan, F C Hou, M Gu. Study on the non-gaussian characteristics and peak factor of across-wind response for super high-rise buildings. Journal of Vibration and Shock, 2020, 39(17): 171-176. (in Chinese)

[16]Z W Wang, B Liu, L J Wang. Finite element verification of nongaussian accelerated random vibration theory based on G_(rms)-N. Chinese Journal of Applied Mechanics, 2020, 37(06): 2386-94+694. (in Chinese)

[17]Y W Su, G Q Huang, Y P Zeng. Research in the effects of buffeting responses of a long-span bridge subjected to weak and strong non-stationary wind events. Journal of Railway Engineering Society, 2019, 36(12): 41-47. (in Chinese)

[18]S Karen, M Sameer, K Rakesh. Application of autocovariance orthogonal decomposition in the response of multi degree of freedom (MDOF) systems to non-stationary random vibration processes[C]. 51st AIAA/ASME/ASCE/AHS/ASC Structures, Structural Dynamics and Materials Conference, April 12, 2010 April 15, 2010.

[19]Capponi, Lorenzo, Česnik, Martin. Non-stationarity index in vibration fatigue: theoretical and experimental research[J]. International Journal of Fatigue, 2017, (104): 221-230.

[20]Palmieri, Massimiliano, Česnik, Martin. Non-gaussianity and non-stationarity in vibration fatigue[J]. International Journal of Fatigue, 2017, (97): 9-19.

[21]Z Yang. Research on fatigue life analysis method of vibration system under non-gaussian random excitation. Lanzhou: Lanzhou Jiaotong University, 2020. (in Chinese)

[22] Yu H, Wang B, Xia C, et al. Efficient non-stationary random vibration analysis of vehicle-bridge system based on an improved explicit time-domain method. Engineering Structures, 2021, 231(111786).

[23]Z Q Hu. Vibration and shock test technology. Beijing: Chinese Standards Press, 2019. (in Chinese)

[24]P Wolfsteiner, A Trapp. Fatigue life due to non-gaussian excitation-an analysis of the fatigue damage spectrum using higher order spectra. International Journal of Fatigue, 2019, (127): 203-216.

[25]V Rouillard. Quantifying the non-stationarity of vehicle vibrations with the run test. Packaging Technology and Science, 2013, (27): 203-219. 
[26]M R Masliah. Stationarity/non-stationarity Identification. Tech rep. Toronto: University of Toronto, 2004.

[27]X Zeng, Y Jiang, Z W Fan, W Y Lei. Characterization and simulation method of non-stationary random vibration in packaging and transportation test. Packaging Engineering, 2021, 42(09): 1-10. (in Chinese)

[28]F Li, H Wu, P Wu. Vibration fatigue dynamic stress simulation under non-stationary state. Mechanical Systems and Signal Processing, 2021, 146(107006)

[29]Q Q Yu, J Zhen, J C Liu, C W Wang. Analysis of test method for damping characteristics of aluminum alloy plate. Electro-Mechanical Engineering, 2012, 28(02): 1-3+7. (in Chinese)

\section{Biographical notes}

Xin Zeng, born in 1991, is currently an engineer at National University of Defense Technology. He received his master degree from National University of Defense Technology, China, in 2021. His research interests include structural vibration fatigue.

Tel: +86-18053865820; E-mail: zengxin18@nudt.edu.cn

Yu Jiang, born in 1977, is currently a professor and a $\mathrm{PhD}$ candidate supervisor at Laboratory of Science and Technology on Integrated Logistics Support, National University of Defense Technology, China. His research interests include structural dynamic reliability, structural vibration fatigue and Structural health monitoring.

Tel: +86-13873190709; E-mail: jiangyu@nudt.edu.cn

Wu-Yang Lei, born in 1997, is currently a master candidate at Laboratory of Science and Technology on Integrated Logistics Support, National University of Defense Technology, China.

E-mail: leiwuyang@nudt.edu.cn

Zheng-Wei Fan, born in 1993, is currently a $\mathrm{PhD}$ candidate at Laboratory of Science and Technology on Integrated Logistics Support, National University of Defense Technology, China.

E-mail: fanzhengwei15@nudt.edu.cn 